\title{
Performance Quantification of a Flat-Panel Imager in Industrial Mega-Voltage X-ray Imaging Systems
}

\author{
Carina Strittt ${ }^{\mathrm{a},}$, Mathieu Plamondon ${ }^{\mathrm{a}}$, Jürgen Hofmann ${ }^{\mathrm{a}}$, Alexander Flisch ${ }^{\mathrm{a}}$, Urs Sennhauser $^{\mathrm{b}}$ \\ ${ }^{a}$ Empa, Swiss Federal Laboratories for Material Science and Technology, Center for X-ray Analytics,Ueberlandstrasse 129, 8600 Dübendorf, Switzerland \\ ${ }^{b}$ Empa, Swiss Federal Laboratories for Material Science and Technology, Reliability Science and Technology Laboratory, Ueberlandstrasse 129, 8600 Dübendorf, \\ Switzerland
}

\begin{abstract}
Active matrix flat-panel detectors have gained popularity amongst X-ray imaging systems due to their speed, resolution and high dynamic range. With appropriate shielding modern flat-panel imagers can even be used in high energy Computed Tomography (CT) systems of energies up to several mega-electronvolt $(\mathrm{MeV})$. However, the performance of a digital detector is not independent of the rest of the radiographic system but depends on all other components of the system. Signal and noise transfer properties highly depend on all parameters of an imaging chain. This work focuses on quantifying the resolution capabilities and the noise in the signals of a MeV X-ray imaging system. The performance quantification is done by computing the modulation transfer function (MTF) using the standard edge method as well as the noise power spectrum (NPS) of the imaging system. We performed Monte Carlo (MC) simulations in order to understand the influence of scattered radiation on the measurements. A comparison of the horizontal and vertical MTF showed that the imaging behaviour of the detector is isotropic. Moreover, an additional investigation of the noise performance of the system showed that there is no measurable noise correlation present in the system. It was shown that the thickness of the edge device does not have a significant influence on the resulting system MTF. A rapid drop in the visibility could be observed resulting in a value of 1.2 line pairs per $\mathrm{mm}$ at 50\% MTF. The visibility limit of line pair patterns was found to be at 2.3 line pairs per mm given by the $10 \%$ MTF value.
\end{abstract}

\section{Introduction}

Detailed knowledge about the signal and noise transfer properties are crucial in understanding the capabilities and limitations of any imaging system. In the field of X-ray imaging as well as X-ray Computed Tomography (CT) these capabilities are typically characterized by the modulation transfer function (MTF) of the detection system as well as its noise power spectrum (NPS). The analyses of accuracies as well as performance characteristics of industrial CT systems have been carried out extensively for micro-CT systems as well as macro-CT systems operating at energies of up to several hundred kilo electronvolt $(\mathrm{keV})[1,2,3]$. However, the energy spectrum of the photons and the atomic number of the scanned material are limiting factors for the penetration length. For heavily absorbing objects, energies exceeding one mega electronvolt $(\mathrm{MeV})$ are needed in order to achieve measurable transmission values. In contrast to conventional X-ray CT systems working at lower energies, which nowadays mostly operate in cone-beam geometry, these high energy X-ray imaging setups are typically designed in a fan-beam configuration employing a line detector which can be highly collimated. The reason for using a fan-beam geometry is that a significant increase in scattered radiation can be observed for high energy X-ray measurements. A highly collimated line detector is able to reduce the scattered radiation in the signal

\footnotetext{
${ }^{*}$ Corresponding author

Email address: carina.stritt@empa.ch (Carina Stritt)
}

significantly [4]. However, the increase in signal quality comes at the price of inspection time. Most commercial flat-panel devices are not designed for the specific task of detecting $\mathrm{MeV} \mathrm{X}$ ray radiation. However, the utilization of a flat-panel imager in a MeV X-ray system is still possible, given a sufficient amount of shielding and accurate source collimation. Nevertheless, the impact of the increase in scattered radiation in the radiographies on the quality of the signal as well as the detailed signal transfer behaviour in this setup has to be analysed.

In this work, we quantify the performance of an industrial MeV $\mathrm{X}$-ray imaging setup employing a flat-panel detector. The quality of the signal as well as the noise propagation in the system will be quantified using the MTF and the NPS. Additional Monte Carlo (MC) simulations will help to quantify the influence of scattered radiation as well as to estimate the contribution of the different system components to the scattered radiation.

\section{Experimental setup}

The setup studied in this work consists of a linear accelerator (see Table $1 \mathrm{~b}$ ) with a focal spot size of $2 \mathrm{~mm}$, which can operate at $\mathrm{X}$-ray energies of 4 and $6 \mathrm{MeV}$. The $\mathrm{X}$-ray beam is generated in a $0.85 \mathrm{~mm}$ thick tungsten target at the end of the linear accelerator. The detector is an active matrix flat-panel imager from Perkin Elmer, model XRD1621, with a pixel size of $200 \mu \mathrm{m}$. 
Tab. 1: Detailed specifications of the components employed in the MeV CBCT setup used in this work.

(a) Specifications of the detector XRD 1621 from Perkin Elmer employed in the system [5].

\begin{tabular}{l|l}
\multicolumn{2}{c}{ XRD 1621 AN14 ES } \\
\hline Manufacturer & Perkin Elmer \\
Pixel number & $2048 \times 2048$ \\
Pixel pitch & $200 \mu \mathrm{m}$ \\
Area & $409.6 \times 409.6 \mathrm{~mm}^{2}$ \\
Scintillator & DRZ-Plus \\
& $208 \mu \mathrm{md}_{2} \mathrm{O}_{2} \mathrm{~S}: \mathrm{Tb}$ \\
Radiation energy & $40 \mathrm{keV}-15 \mathrm{MeV}$
\end{tabular}

The entrance window of the detector consist of a $0.75 \mathrm{~mm}$ aluminium plate. The scintillating medium is a $208 \mu \mathrm{m}$ thick terbium doped gadolinium oxysulfide $\left(\mathrm{Gd}_{2} \mathrm{O}_{2} \mathrm{~S}: \mathrm{Tb}\right)$, which is on top of an amorphous silicon array connected to thin film transistors. The distance between the source and the detector was chosen to be at a maximum of $4.5 \mathrm{~m}$ in order to reduce the influence of the large focal spot of the source on the performance measurements of the detector. For the shielding of the detector, the detection area was reduced by $25 \mathrm{~mm}$ at each side to a size of $359.6 \mathrm{~mm} \times 359.6 \mathrm{~mm}$, which corresponds to a pixel matrix of $1798 \times 1798$ pixel. A $60 \mathrm{~mm}$ thick lead shielding was chosen in order to protect the electronics of the detector from the $\mathrm{X}$-ray radiation. Detailed specifications about the source and the detector can be found in Table 1.

\section{Methods}

\subsection{Noise power spectrum}

The noise in the images is an evident factor, when it comes to analyzing an X-ray imaging systems performance. A good (b) Specifications of the linear accelerator PSL-6D from U.S. Photon Service employed in the system.

Pulstar Linac - PSL-6D

\begin{tabular}{l|l}
\hline Manufacturer & U.S. Photon Service Inc. \\
Linac Design & $2998 \mathrm{MHz}$ (S-Band) \\
Electron Beam Energy & $4 \mathrm{MeV}$ and $6 \mathrm{MeV}$ \\
Maximum Dose Rates & $4 \mathrm{~Gy} / \mathrm{min}$ at $4 \mathrm{MeV}$ \\
at $1 \mathrm{~m}$ distance & $8 \mathrm{~Gy} / \mathrm{min}$ at $6 \mathrm{MeV}$ \\
Target material & Tungsten \\
Focal spot size & $2.0 \mathrm{~mm}$
\end{tabular}

method to quantify the noise in radiographic images is the computation of the noise power spectrum (NPS). Compared to other measures such as the variance or standard deviation, the NPS does not only describe the noise content in the images, but gives information about the spatial frequency behaviour of the noise in the detector. This means that the integrated NPS gives rise to the noise variance. The NPS can be determined from a set of uniformly exposed images as described in [6]. We measured five flat field images. The acquisition parameters of the linear accelerator X-ray source as well as the detector used for the NPS measurements can be found in Table 2. In each flat field image a set of $7 \times 7$ half-overlapping regions of interest (ROI) of size $256 \times 256$ pixel were determined. Each of the ROIs is detrended in order to remove low frequency, large scale inconsistencies which can be a result of varying exposure levels throughout the image. The removal is done by fitting a two dimensional second order polynomial $P_{2}$ to the flat-field image and subsequently subtracting it. The resulting NPS for the horizontal and vertical spatial frequencies $u_{n}$ and $v_{k}$ is computed as given in equation 1 .

$$
\begin{aligned}
& \text { NPS }\left(u_{n}, v_{k}\right)=\frac{d x \cdot d y}{M \cdot 256 \cdot 256} \sum_{m=1}^{M}\left|\sum_{i=1}^{256} \sum_{j=1}^{256} \tilde{I}_{m}\left(x_{i}, y_{j}\right) \exp \left(-2 \pi i\left(u_{n} x_{i}+v_{k} y_{j}\right)\right)\right|^{2} \\
&(d x, d y) \text { Pixel spacing in horizontal and vertical direction } \\
& M \text { Total number of ROIs } \\
&\left(u_{n}, v_{k}\right) \text { Horizontal and vertical spatial frequencies } \\
&(x, y) \text { Pixel locations } \\
& \tilde{I}_{m}(x, y)=I_{m}(x, y)-P_{2}(x, y), \text { detrended ROI } \\
& P_{2}(x, y) \text { Two dimensional second order polynomial fit }
\end{aligned}
$$

\subsection{Modulation transfer function}

The modulation transfer function (MTF) measures the transfer of contrast in the system by comparing the real contrast of the measured object to the output contrast of the detection system.
It is commonly plotted as a function of the spatial frequency, referring to the ability of the system to image a black and white line pattern of a given frequency. There are several test objects that can be chosen to measure the MTF of an X-ray imaging 
Tab. 2: Acquistion parameters of the NPS measurements.

\begin{tabular}{|c|c|c|}
\hline \multicolumn{3}{|c|}{ X-ray Source - Pulstar Linac PSL-6D } \\
\hline Energy & {$[\mathrm{MeV}]$} & 6.0 \\
\hline Frequency & [pps] & 122 \\
\hline Dose rate at $1 \mathrm{~m}$ distance & {$[\mathrm{mGy} / \mathrm{s}]$} & 91.7 \\
\hline \multicolumn{3}{|c|}{ Detector - XRD 1621 AN14 ES } \\
\hline Gain & {$[\mathrm{pF}]$} & 0.5 \\
\hline Integration time & [ms] & 1568 \\
\hline Binning mode & & no binning \\
\hline
\end{tabular}

system. The ones most frequently used are a bar pattern object, a slit or an edge [7]. In this work the edge method was chosen for the determination of the MTF of the imaging system. An edge test object has the form of a plate, which is favourably constructed with a sharp edge. Moreover, it should be made of a dense material thick enough to block all incident X-ray radiation. It is clear that in the case of a mega-voltage X-ray CT system satisfying both of these conditions at the same time is not possible. An opaque edge would require a thick layer of material and thus negate the sharp edge requirement. This means that the only option for measuring the MTF with an edge method in a high energy X-ray system is a semi-transparent version of the edge method. As a result, the trade-off between the sharpness of the edge of the object and the contrast in the image has to be examined. To investigate the influence of the thickness of the edge object on the calculated MTF, the edge measurements were performed using four tungsten plates with thicknesses of 1, 2, 5 and $10 \mathrm{~mm}$. A list of the whole parameter space covered in the measurements of the MTF can be found in Table 3. Each plate was placed directly in front of the entrance window of the flat-panel detector. The acquisition parameters of the detector were kept the same as for the NPS measurements and can be found in table 2. The MTF measurements were performed at both energy settings of the linear accelerator, namely 4 and $6 \mathrm{MeV}$ with a frequency of 122 pps (pulses per second). The corresponding dose rates at $1 \mathrm{~m}$ distance to the source are $57.9 \mathrm{mGy} / \mathrm{s}$ and $91.7 \mathrm{mGy} / \mathrm{s}$ respectively. The edge was arranged at a slight angle of about $1.5^{\circ}$ with respect to the image axis to rule out an influence of the detector matrix on the measurement. The edge spread function (ESF) is then extracted perpendicular to the edge of the object. Here, the ESF was extracted for all four test objects at both beam energies in horizontal and vertical direction with respect to the flat-panel axis. From the ESF, the line spread function (LSF) can be calculated by differentiation. The resulting LSF is smoothed using a gaussian fit and additionally normalized. Finally, the MTF can be determined by performing a Fourier transform on the LSF.

Even though it is not possible to construct the perfect edge device for the high energy application, the edge method cho- sen here is still favourable compared to the other two common methods of measuring the MTF of an X-ray imaging setup. A measurement of the MTF with a bar-pattern test object, while certainly the most straightforward of the three options, can only return discrete values and the continuous MTF has to be approximated by interpolation [7]. In contrast to the bar-pattern method, the measurement of the MTF using a slit object, is able to yield a continous MTF. However, the method is arguably more complicated to measure since it requires a good alignment of the slit with the source. Moreover, the slit method has often times shown to yield noisy tails in the LSF [7].

Tab. 3: Configurations used for the MTF measurements.

\begin{tabular}{lll}
\hline Parameter & Unit & Value \\
\hline Thickness & {$[\mathrm{mm}]$} & $\{1.0,2.0,5.0,10.0\}$ \\
Energy & {$[\mathrm{MeV}]$} & $\{4.0,6.0\}$ \\
Direction & & $\{$ horizontal, vertical $\}$ \\
\hline
\end{tabular}

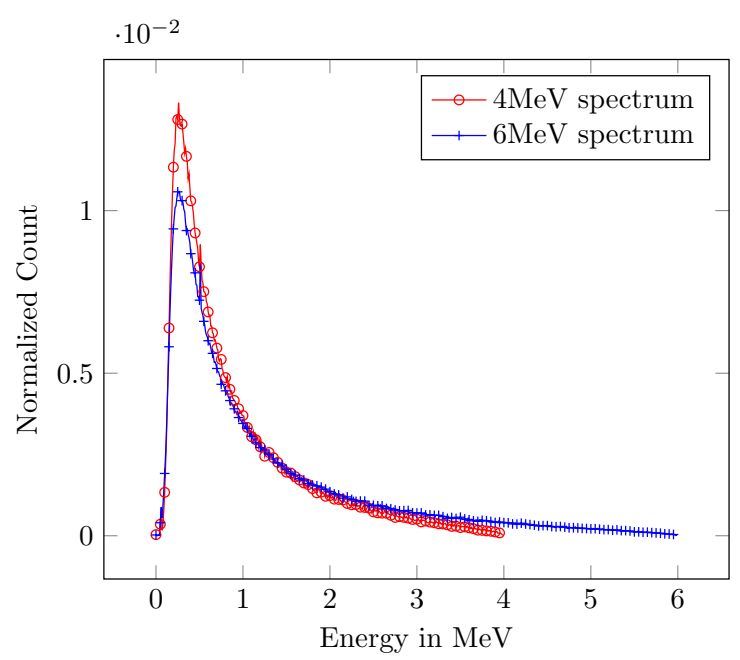

Fig. 1: The pre-simulated spectra used for the simulations. The spectra were created in MC simulations performed in GEANT4. For the simulations of the spectrum a mono-energetic electron pencil beam of was shot onto a $0.85 \mathrm{~mm}$ thick tungsten target. Resulting photons were recorded in $1 \mathrm{~m}$ distance to the target in an area covering a $2.5^{\circ}$ half-angle with respect to the source.

\subsection{Monte Carlo Simulations}

Additionally to the measurements, we performed Monte Carlo (MC) simulations of the MeV X-ray imaging setup to understand the relative influence of the different scatter effects and system components on the edge signal. A popular framework in the context of particle simulations is GEANT4 [8]. GEANT4 enables the tracking and monitoring of all physical interactions as well as the implementation of a realistic model of the CT setup. For the simulations in this work, version 4.9 .6 of the framework was used. As data basis for the physical processes, the low energy electromagnetic package Penelope (PENetration and Energy LOss of Positrons and Electrons) was applied. The 


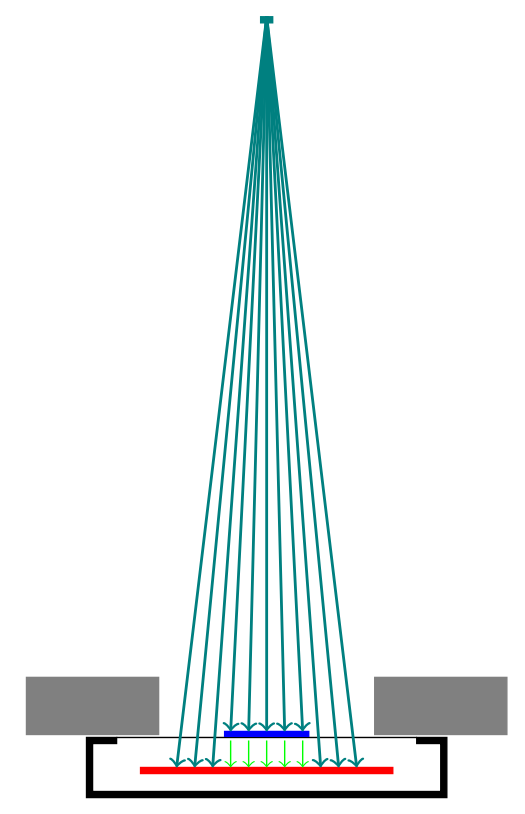

(a) Sketch of the system setup used in the Monte Carlo Simulations

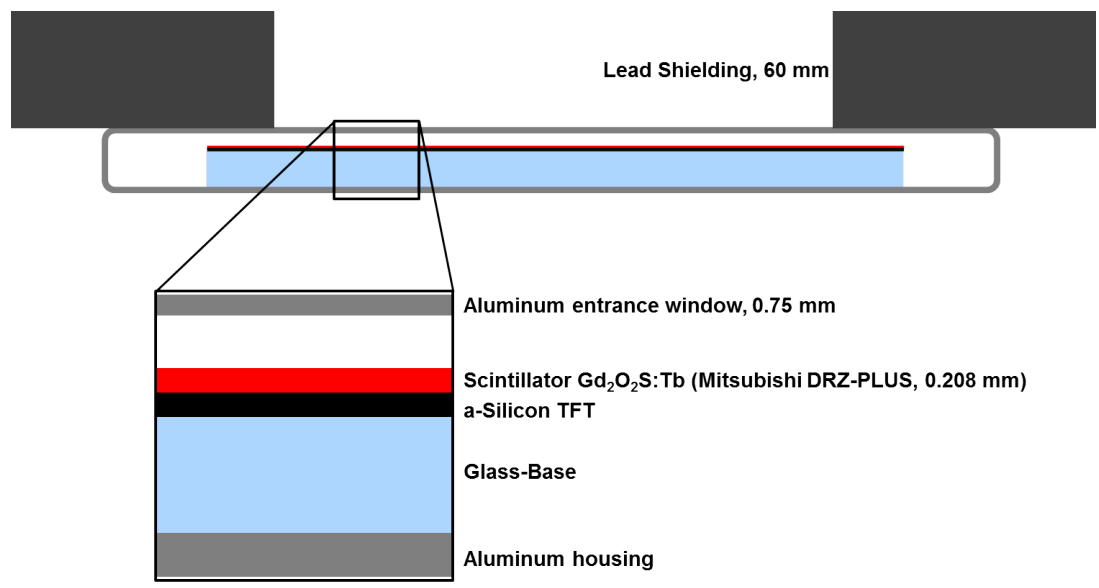

(b) Sketch of the model of the detector used for the simulations with the additional lead shielding.

Fig. 2: System setup and detailed information about the detector used in the Monte Carlo simulations described in section 3.3. Distances, sizes as well as the placement of the edge device are given in section 2. Figure 2a) Sketch of the system setup. The distances between the source and detector were chosen in accordance with the experimental setup. The edge device (in blue) is placed directly in front of the detectors entrance window. Figure $2 b$ ) Detailed sketch of the detector as it was modelled in the simulation. Impinging radiation is recorded when entering the scintillator (in red). The close-up shows the structure of the detector incorporating the scintillator as well as a thin film transistor on a glass base.

considered physical interactions include the photoelectric effect, Compton scattering, pair production and Rayleigh scattering for the photons and multiple scattering, ionization, bremsstrahlung and annihilation for the electrons and positrons. For performance purposes the simulation of optical photons in the scintillator has been omitted. All simulated system components were modelled as native GEANT4 geometrical primitives using material properties from the NIST database on atomic weights and isotope compositions provided by GEANT4. The model used for the simulation of the detector can be seen in Figure 2. Included in the simulation are the lead shielding, an aluminum entrance window, the detector housing as well as the scintillating medium of the detector. The source to detector distance as well as the simulated edge object matched the parameters of the measurements described previously. The finite sized focal spot of the X-ray source is modelled as a disk with radius $1 \mathrm{~mm}$. The photons are emitted randomly from this disk with a direction which is sampled randomly from a cone-beam isotropic distribution with a half-angle of $2.5^{\circ}$. The spectrum of the linear accelerator source was simulated separately by using a beam of monochromatic electrons impinging on a $0.85 \mathrm{~mm}$ thick tungsten target. Detailed information about the source simulations can be found in [4]. The spectrum used for the simulations at the detector response can be seen in Figure 1. To obtain sufficient photon statistics on the detector, we perform ten runs of the simulation in parallel, with different random seeds and $10^{7}$ photon histories each. For each photon we record the energy and its scattering history. The photons were categorized in two ways. The order of scattering was recorded distinguishing primaries, single and multiple scattered photons and the scattering volumes were identified and matched with the photons. This enables us to investigate the impact of scattered radiation on the signal as well as the influence of all simulated system components on the scatter statistic.

\section{Results}

\subsection{Noise power spectrum}

Figure 3 a shows the two dimensional NPS for the horizontal and vertical spatial frequencies up to 3 cycles per $\mathrm{mm}$. It can be seen that the distribution of the NPS is radially symmetric with respect to the origin at spatial frequency $(0,0)$. According to the procedure in [6] a total of 14 rows around each axis in the 2D NPS (excluding the axis itself) were extracted and averaged as well as normalized to the so-called squared large area signal, which is given as the square of the mean signal of the flat fields. The resulting vertical and horizontal NPS (see Figure 3b) do not show any significant differences. The broadness of these normalized NPS (NNPS) shows that the noise in the images is not correlated. This stems from the fact that uncorrelated noise leads to a peaked autocorrelation function, which subsequently turns into the broad NNPS, whereas correlated noise would have the exact reverse effect of generating a broad autocorrelation function which in turns leads to a peaked NPS (see [7]). This means that we can expect the measured radiographies not to exhibit a spatial pattern of noise. 


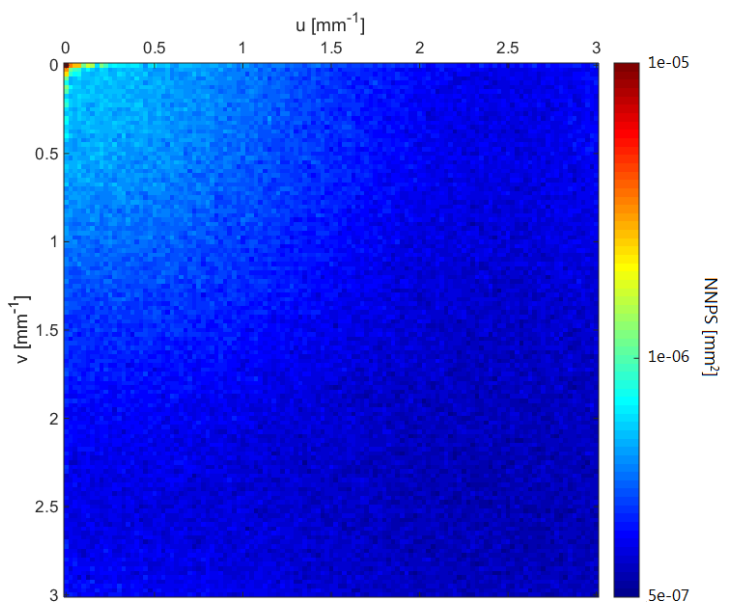

(a) Plot of the two dimensional NPS.

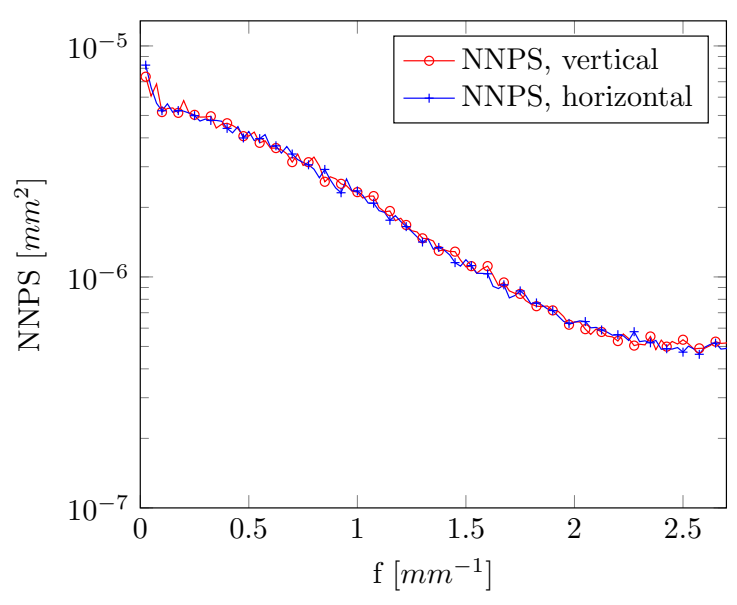

(b) Plot of the horizontal and vertical normalized NPS.

Fig. 3: The noise power spectrum of the flat panel detector. Figure 3a) shows the two dimensional NPS for the spatial frequencies u and v. The horizontal and vertical NPS in Figure 3b) were normalized with the squared large area signal and computed from the two dimensional NPS according to the procedure in [6].

\subsection{Modulation transfer function}

As described previously, for the determination of the MTF with the edge method, the edge spread function (ESF) perpendicular to the edge of the objects has to be determined. An example of the measurement of the $2 \mathrm{~mm}$ edge device can be seen in Figure 4a. All edge devices were measured at both energies as well as in horizontal and vertical direction. Figure $4 \mathrm{~b}$ shows the results for the $2 \mathrm{~mm}$ edge. No significant difference between the measurements applied to the horizontal and vertical edge can be found, which means that we can assume the MTF to be isotropic. It is clearly visible that the measured edge objects are semitransparent to the incident X-ray beam, which means that there is a non-zero contribution to the signal from transmission X-rays at the position of the object in the image. The transparency of the four edge objects ranges from about $40 \%$ up to $88 \%$ for the thinnest edge object. This is a result of the MeV Xray imaging setup itself. An opaque edge object, which would block the beam itself, in this configuration, would need to be significantly thicker than the $1 \mathrm{~mm}-10 \mathrm{~mm}$ edge devices used in these measurements. However, the authors decided against the use of thicker objects as it would be almost impossible to align with the beam and thus negate the concept of a sharp edge in the image.

It is interesting to notice in Figure $4 b$ that the edge signal exhibits a negative and positive excess right before and after the edge. These jumps in the signal can be seen for all of the four edge devices.

A first hypothesis on the origin of this effect can be a misalignment of the edge object with the beam and a subsequent reflection of the X-rays. Due to the cone-beam setup, only incident radiation in the center of the detector will have a direction that is normal with respect to the detector surface. A misplacement of the edge with respect to this center can result in incoming radiation hitting the side of the object as opposed to the ideal case where the incident beam only hits the front of the object. This in turn can lead to X-rays scattering away from the objects side resulting in an enhancement of the signal in air close to the edge. A sketch of the effect of reflected radiation from a misaligned edge can be seen in Figure 5a. Another hypothesis on the cause of this effect might be scattered radiation within the object. In this case, the combination of the transmitted signal of the object and the varied signal of the scattered radiation would create this negative and positive jump in the edge spread function. The reason for the magnitude of the effect is primarily the proximity between the edge object and the scintillator. Moreover at the high energies used in the measurements single scattered radiation will be highly forward directed. At a pixel at location $i$ corresponding to the middle of the object the measured value will consist of the transmitted primary radiation combined with scattered radiation from all directions surrounding the pixel. Closer to the edges of the object, the contribution of scattered radiation from one side will diminish. This means the factor $I_{s}(n)$ in equation 2 will decrease simply due to a decrease in the amount of neighbourhood locations $N$ of the object. As a result the overall measured signal $I(i)$ will decrease with decreasing distance to the edge.

$$
\begin{aligned}
& I(i)=I_{p}(i)+\sum_{n \in N} I_{s}(n) \\
& \text { I(i) Measured pixel value at location } i \\
& I_{p}(i) \quad \text { Primary signal at } i \\
& N \quad \text { Neighbourhood of the pixel location } \\
& \text { at the object } \\
& I_{s}(n) \quad \text { Scattered radiation from } n
\end{aligned}
$$

Likewise, the flat field value measured in a large distance to the object, can be assumed to consist only of the air value of the image without any contributions from object scattered radiation due to the proximity between object and scintillator. The pixel value in the air close to the edge of the object on the other hand will contain both the flat field signal plus an additional signal 


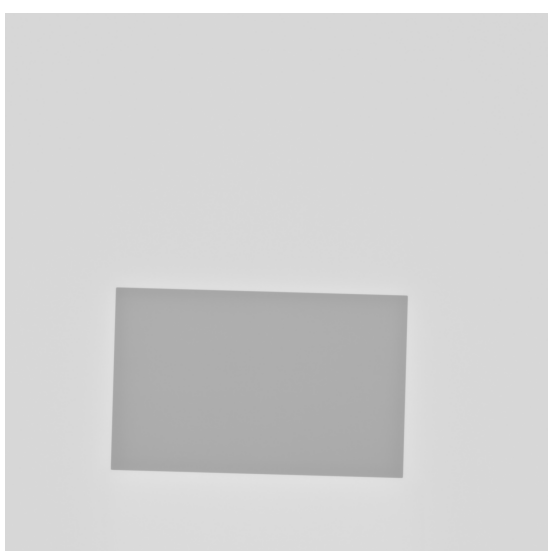

(a) Edge measurement, $2 \mathrm{~mm}$ tungsten plate.

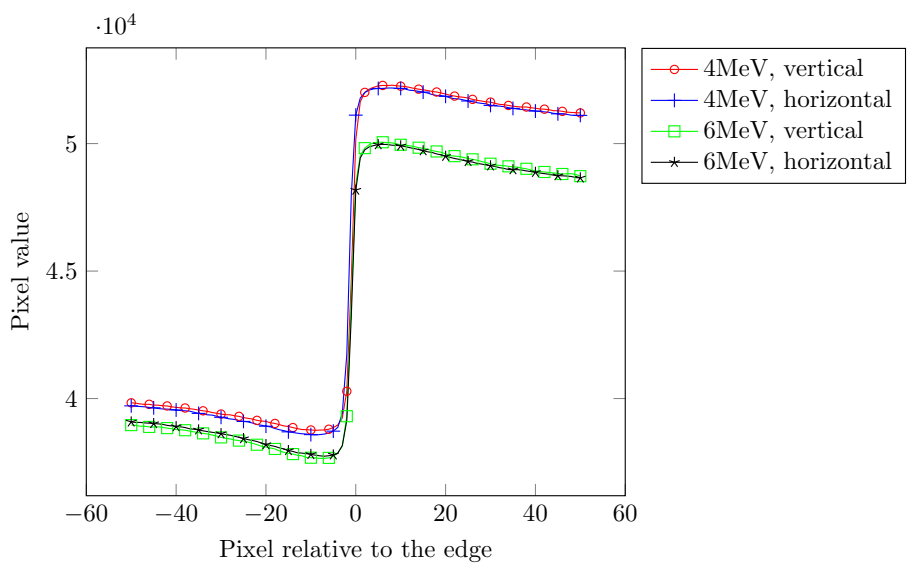

(b) Edge spread function, $2 \mathrm{~mm}$ edge object.

Fig. 4: Examples of the edge measurements as well as the extracted edge spread functions for the $2 \mathrm{~mm}$ edge object. Figure $4 \mathrm{a}$ ) Edge measurement for the $2 \mathrm{~mm}$ edge object measured with the $6 \mathrm{MeV}$ spectrum for the extraction of the horizontal edge spread function. As it can be seen the horizontal edge is placed at a slight angle with respect to the image axis in order to rule out an influence of the detector matrix on the measurement. Figure 4b) Plot of the edge spread function (ESF) for the $2 \mathrm{~mm}$ edge object averaged over 100 pixels along the edge. Results for the measurements at both energies $4 \mathrm{MeV}$ and $6 \mathrm{MeV}$ and both directions are shown.

resulting from object scattered radiation. A sketch of this process can be seen in Figure 5b. The MC simulations performed in this work, discussed in section 4.3 aim to investigate these concepts in more detail.

From the ESF, the line spread function (LSF) can be calculated by differentiation. Additionally, the resulting LSF was smoothed using a gaussian fit and normalized such that its integral is one. Figure 6 shows the LSF in the exemplary case of the $1 \mathrm{~mm}$ edge object in horizontal direction.

The MTF can now be determined by performing a Fourier transform on this LSF. The resulting MTF for both the horizontal and vertical direction as well as both energies can be seen in Figure 7. It is interesting to notice that the MTFs of all edge objects match to a high degree. Slight differences can only be detected in the case of the vertical MTF measurements. These differences most likely stem from a misplacement of the edge with respect to the center of the detector as well as the incident beam. The impact of a misaligned edge object with the cone beam on the ESF can be seen in Figure 8. Generally, the results for the thicker edge objects seem to suggest a larger drop in the MTF and thus a higher blur in signal transmission. This can be attributed to the fact that the thicker the edge object is, the more difficult an alignment between the beam and the edge object becomes. Figure 8 shows, that due to the cone-beam shape of the beam a large misalignment can have the effect of a less pronounced edge spread function. This effect can be avoided by choosing a sharper edge device. For these measurements however, a trade-off between contrast and edge size limits the sharpness of the object. Another way to reduce the influence of the cone shape of the beam is to increase the distance between the source and the detector. A larger source to detector distance will render the incident beam on the detector more parallel.

The resulting MTFs can now be interpreted as a form of system blur with respect to the investigated spatial frequencies. In order to make a statement about the resolution capabilities of the system the means of the resulting MTFs of all measurements given in Table 3 were calculated. Table 4 shows a selection of MTF values with their respective resolution given in line pairs per $\mathrm{mm}$. It can be seen that the visibility drops quite rapidly resulting in a value of 1.2 line pairs per mm at $50 \%$ MTF. The theoretical limit of the resolution of a detector with pixel size of $200 \mu \mathrm{m}$ is given by

$$
f=\frac{1 \text { line pair }}{2 \cdot \text { pixel size }}=2.5 \text { line pairs per } \mathrm{mm} \text {. }
$$

However, Figure 7 shows that the visibility of line pair patterns is below this theoretical limit. Typically the resolution limit of a system for which details in the output of the imaging system can no longer be considered to be recognizable is given at a MTF value of 0.1 . In this case the MTF value of $10 \%$ corresponds to a limiting spatial frequency of 2.3 line pairs per $\mathrm{mm}$.

Tab. 4: List of MTF values with their respective spatial frequency given in line pairs per $\mathrm{mm}$.

\begin{tabular}{cc}
\hline MTF [\%] & Resolution [lp/mm] \\
\hline 50 & 1.2 \\
25 & 1.7 \\
20 & 1.8 \\
10 & 2.3 \\
\hline
\end{tabular}

\subsection{Monte Carlo simulations}

In order to validate the hypothesis made earlier in this work concerning the influence of scattered radiation on the edge signal (see section 4.2), MC simulations of all configurations given 


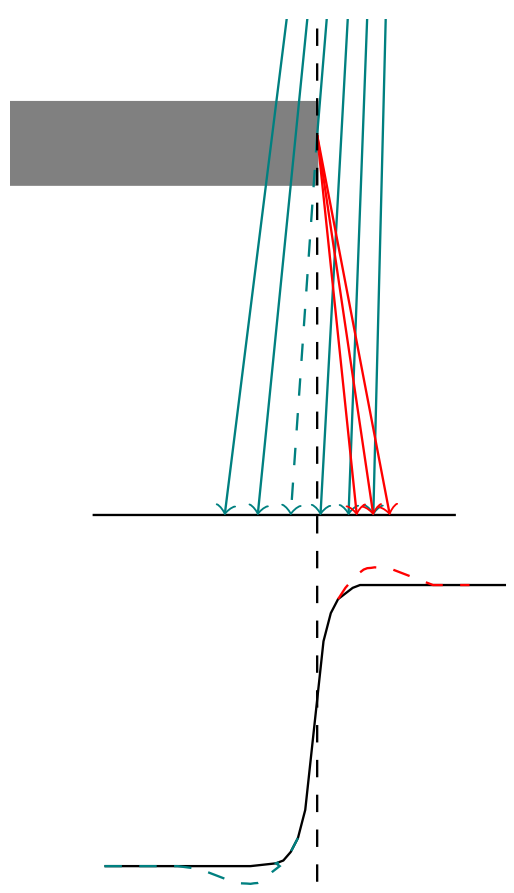

(a) Sketch of the effect of misalignment on the ESF.
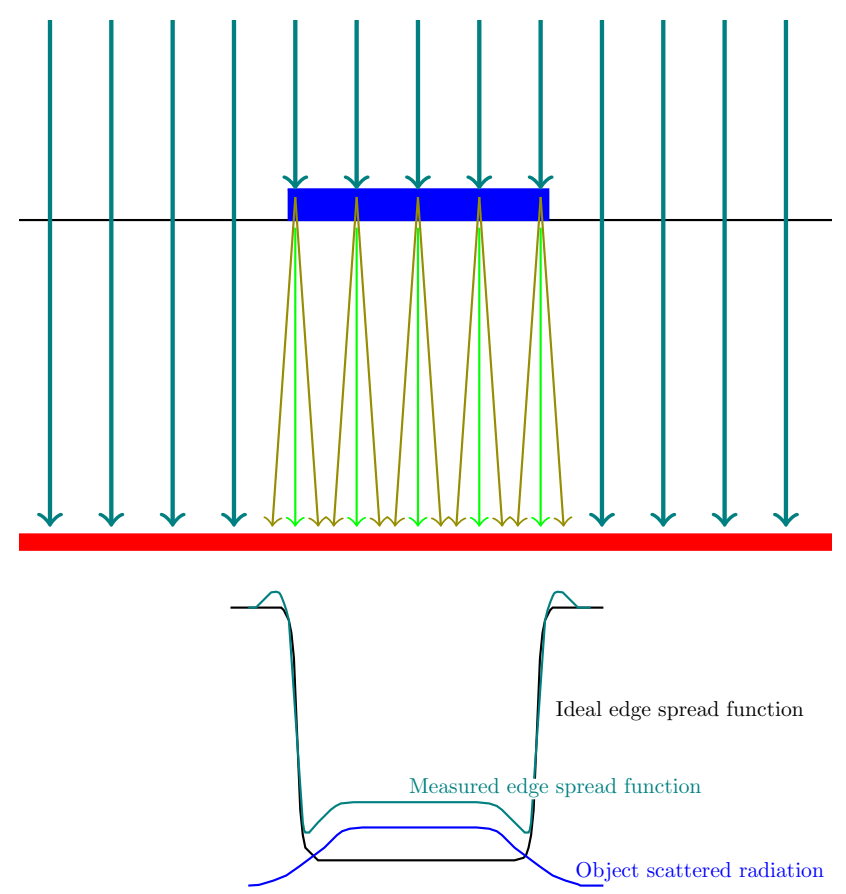

(b) Sketch of the effect of object scattering on the ESF.

Fig. 5: Sketches of the two hypotheses for the overshoot observations in the ESF. Figure 5a shows the effect of reflected photons on the ESF due to a misalignment of the edge object with the beam. Figure $5 \mathrm{~b}$ shows the effect of transmitted object scattered radiation as described in Equation 2.

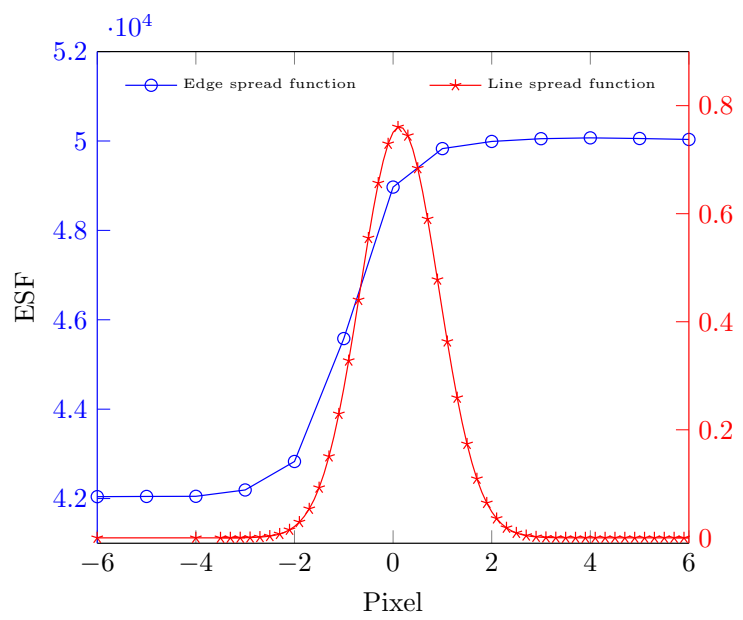

Fig. 6: The edge spread function (ESF) with the corresponding line spread function (LSF) for $1 \mathrm{~mm}$ edge object.

in Table 3 have been performed. The MC simulations allow us to analyse the detected radiation in a detailed manner. Particularly, the photons can be classified with respect to their order of scattering as well as the volume which caused to the scattering. These properties as well as the energy and position of the photons are recorded when entering the scintillating medium. The case of the simulated edge spread function for the $10 \mathrm{~mm}$ edge device can be seen in Figure 9. The simulations were able to recreate the overshoot seen in the measurements (see Figure 4). Due to the fact that a misalignment of the object can be avoided in the case of a simulation the occurence of the nega- tive and positive jump in this case confirms our assumption that the jump in the signal can not solely be attributed to reflected photons from the objects edge. It is interesting to notice that scattered photons can be detected over the whole pixel range, however, the scattered to primary ratio is significantly enhanced for the pixels corresponding to transmission values at the edge object. This non-zero contribution of scattered radiation to the signal corresponding to the attenuated beam as well as the air can be explained by added scattering from the imaging system itself. In addition to the scattering of the object, the entrance window of the detector as well as detector internal components such as the glass base can create scattered radiation. Figure 10 shows the classification of scattered radiation with respect to all these sources of scattered radiation. The close-ups in Figure $10 \mathrm{~b}$ and $10 \mathrm{c}$ show that object scattered radiation is confined to the left-side of the signal and drops down to a zero-contribution to the air signal at a larger distance with respect to the edge of the object. This can of course be attributed to the high proximity between the object and the scintillator. For typical measurements at larger object to detector distances the contribution of object scattered radiation will distribute over a larger area at the detector. The other two components of the scattered radiation that were analysed here, the detector internal scattering and the entrance window scattering, dominate the total scattered radiation on the right hand side of the signal. A slight decrease of their contribution can be seen for the attenuated beam. Scattered radiation resulting from the shielding of the detector (see Figure 2) was not accounted for in this simulation as it only has a local effect on the radiographies at the image margins 


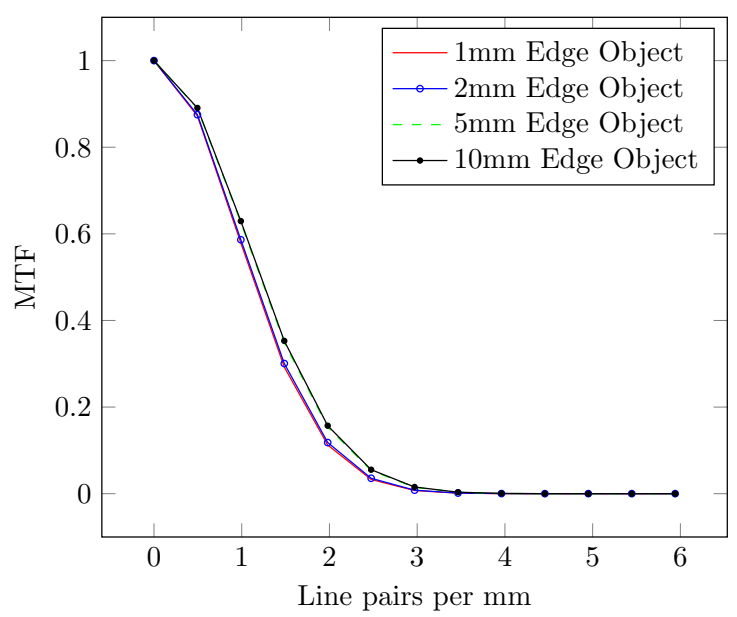

(a) MTF for an energy of $4 \mathrm{MeV}$ in horizontal direction.

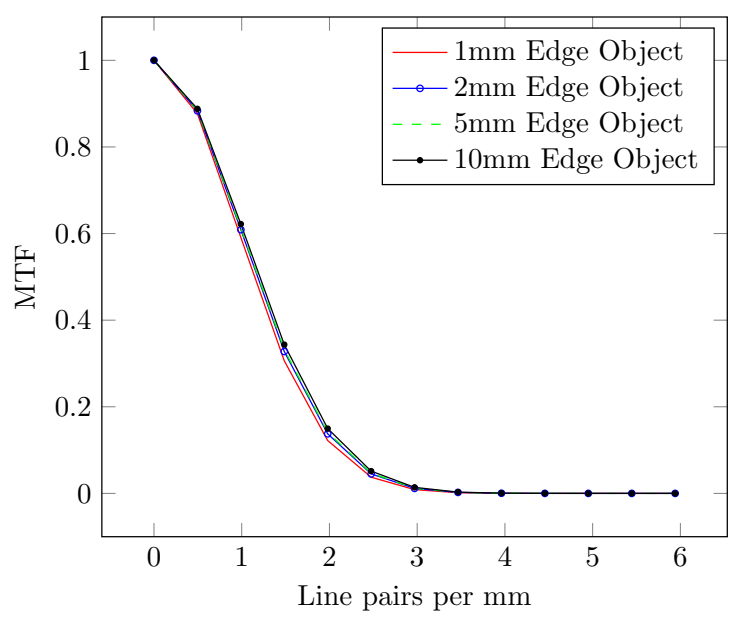

(c) MTF for an energy of $6 \mathrm{MeV}$ in horizontal direction.

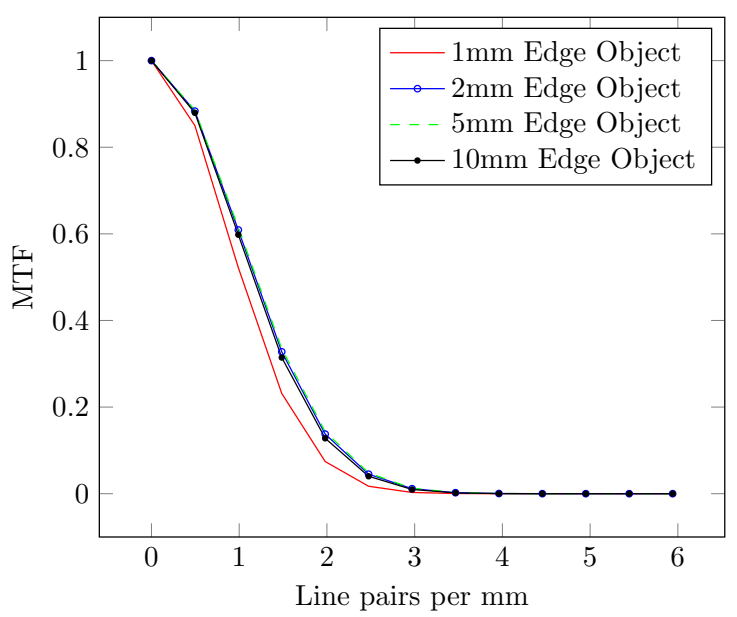

(b) MTF for an energy of $4 \mathrm{MeV}$ in vertical direction.

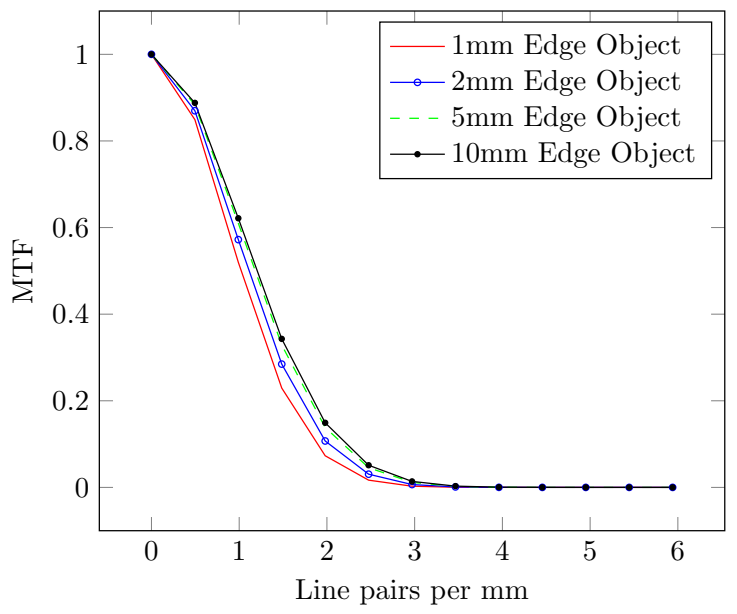

(d) MTF for an energy of $6 \mathrm{MeV}$ in vertical direction.

Fig. 7: Modulation transfer function for all configurations given in Table 3. The MTFs are plotted versus the spatial frequency given in line pairs per mm.

(see [9]). It is worth noticing that a given multiple scattered photon can account for two or even more of the recorded categories which means that the sum of all scatter components of the system scattered radiation will not represent the total scattered radiation because of multi-counting of the same photons. However, this effect will be small as single scattered photons dominate over multiple scattered photons in this case. It should be noted that a significant decrease in object scattered radiation can be assumed for increased object to detector distances [9].

\subsection{Comparison to other MTF measurements}

Other MTF measurements on amorphous silicon flat-panel detectors generally measure the MTF of the X-ray imaging system using the radiation qualities (RQA) outlined in the international standard for the characterisation of digital X-ray imaging devices [6]. This means, the measurements are restricted to X-ray tube voltages from tens of $\mathrm{keV}$ up to several hundred $\mathrm{keV}$. The measurements in this work were performed at X-ray beam energies that are an order of magnitude higher then the values given in the radiation quality standards.
Exemplary cases of MTF measurements using the edge technique and an amorphous silicon flat panel detector with a similar pixel pitch to the one used in this work are [10, 11]. These measurements were performed at X-ray beam spectra complying with RQA5 [6], which means they were conducted at Xray beam energies of about $70 \mathrm{keV}$. The results show that in these low energy X-ray applications the MTF doesn't drop below $20 \%$ at 2.5 line pairs per mm, whereas the MTF in the high energy X-ray measurements performed in this work already reaches the visibility limit at this resolution.

Comparisons have to be handled with care, however. Not only beam energies, but also the type of the scintillator in the detector has a big impact on resolution capabilities. The two main scintillator materials in use are caesium iodide (CsI) and gadolinium oxysulfide (Gadox). While CsI scintillators generally have to be used at lower radiation doses, they also entail a structured scintillator crystal arrangement, providing higher resolution capabilities compared to powder scintillators such as Gadox. 


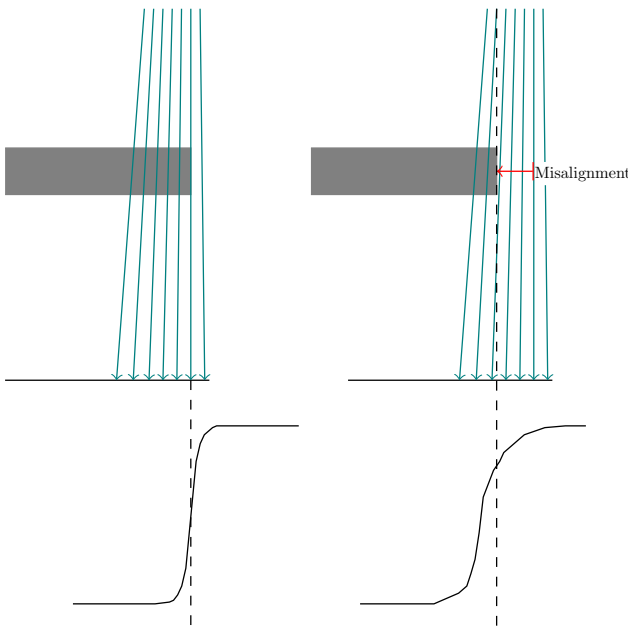

Fig. 8: Sketch of the influence of a misalignment of an edge object with the cone beam to the edge spread function. Due to the cone-beam shape of the beam a large misalignment can have the effect of a less pronounced edge spread function.

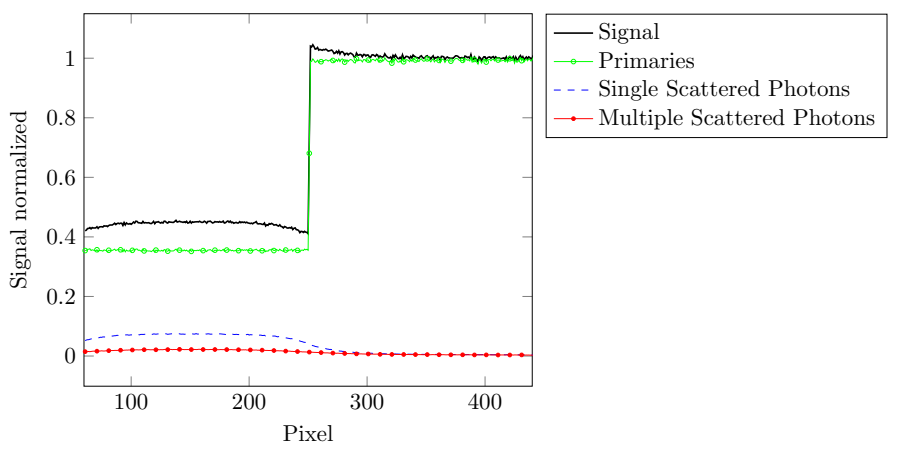

Fig. 9: Results of the simulation of the edge spread function for the $10 \mathrm{~mm}$ edge object. The recorded photons were classified corresponding to their order of scattering.

Moreover, it can clearly be seen in this work, that the higher $\mathrm{X}$-ray energies introduce the problem of X-ray transmission on the edge measurments. The semi-transparent edge objects do not satisfy the international standard on the measurement of the characteristics of digital X-ray imaging devices, which advices opaque edges for the MTF measurements [6]. However, semitransparent edge measurements are not uncommon and have been used before in the context of MTF determination [12]. The X-ray transmission connected to the thin edge object has the disadvantage of introducing scatter into the measurement. Nevertheless, the measurements in this work, comparing different edge object thicknesses, show that the level of transparency doesn't seem to have a measurable influence on the final MTF result.

\section{Discussion}

Measurements and simulations of the signal and noise performance of a MeV X-ray imaging system incorporating an active matrix flat panel imager have been performed. To quantify the signal transfer properties, the NPS and the MTF have been determined. The two dimensional NPS as well as the extracted one dimensional NPS showed that there is no correlated noise present in the system. For the MTF measurements the edge method was chosen and subsequently tested with 4 different tungsten edge devices ranging from a thickness of $1 \mathrm{~mm}$ to a maximum thickness of $10 \mathrm{~mm}$. The comparison of measurements in horizontal and vertical direction proved the isotropic behaviour of the MTF. Despite the fact that none of the four edge devices were able to fully block the incident X-ray beam, an MTF could be deduced from the measurements. Moreover, no significant difference in the results could be found for the different edge devices, which means that all of the edge objects are equivalent for the measurements. However, it could be seen that the semi-transparent objects introduce scattered radiation into the edge spread function. The overshoot artefact which could be observed in the edge spread function could be traced back to scattered radiation using MC simulations. Additionally, the simulations showed that the overshoot resulted mainly from radiation scattered in the object itself. Overall, the MTF of the system was deduced to be at $14.6 \%$ at a spatial frequency of 2 line pairs per $\mathrm{mm}$. Moreover, in this case the general visibility of line pair patterns has a cut off at 2.3 line pairs per mm which lies below the theoretical limit of 2.5 line pairs per $\mathrm{mm}$.

\section{Acknowledgement}

This work is part of the project "X-ray 3D Computed Tomography with Mega Electronvolt Source" (CTOMES). The authors would like to thank the Swiss National Science Foundation (SNF) and the German Research Foundation (DFG) for funding.

\section{References}

[1] M. Bartscher, U. Hilpert, J. Goebbels, G. Weidemann, Enhancement and proof of accuracy of industrial computed tomography (ct) measurements, CIRP Annals-Manufacturing Technology 56 (1) (2007) 495-498.

[2] J. Hiller, M. Maisl, L. M. Reindl, Physical characterization and performance evaluation of an $\mathrm{X}$-ray micro-computed tomography system for dimensional metrology applications, Measurement Science and Technology 23 (8) (2012) 085404.

[3] J. Rueckel, M. Stockmar, F. Pfeiffer, J. Herzen, Spatial resolution characterization of a X-ray microct system, Applied Radiation and Isotopes 94 (2014) 230-234.

[4] C. Stritt, P. Schütz, J. Hofmann, A. Flisch, U. Sennhauser, Quantitative assessment of scattering contributions in $\mathrm{MeV}$-industrial x-ray computed tomography, Proceedings of the 11th European Conference on NonDestructive Testing.

[5] PerkinElmer, XRD $1621 \mathrm{xN}$ ES product note, http://www . perkinelmer.de/ (2016).

[6] I.-I. E. Commission, et al., Medical electrical equipment-characteristics of digital x-ray imaging devices-part 1: Determination of the detective quantum efficiency, iec 62220-1, Geneva, Switzerland.

[7] E. Samei, Performance of digital radiographic detectors: quantification and assessment methods, Advances in digital radiography: RSNA (2003) 37-47.

[8] S. Agostinelli, et al., GEANT4 - A simulation toolkit, Nuclear Instruments and Methods in Physics Research, Section A: Accelerators, Spectrometers, Detectors and Associated Equipment 506 (3) (2003) 250-303. doi:10.1016/S0168-9002(03)01368-8. 


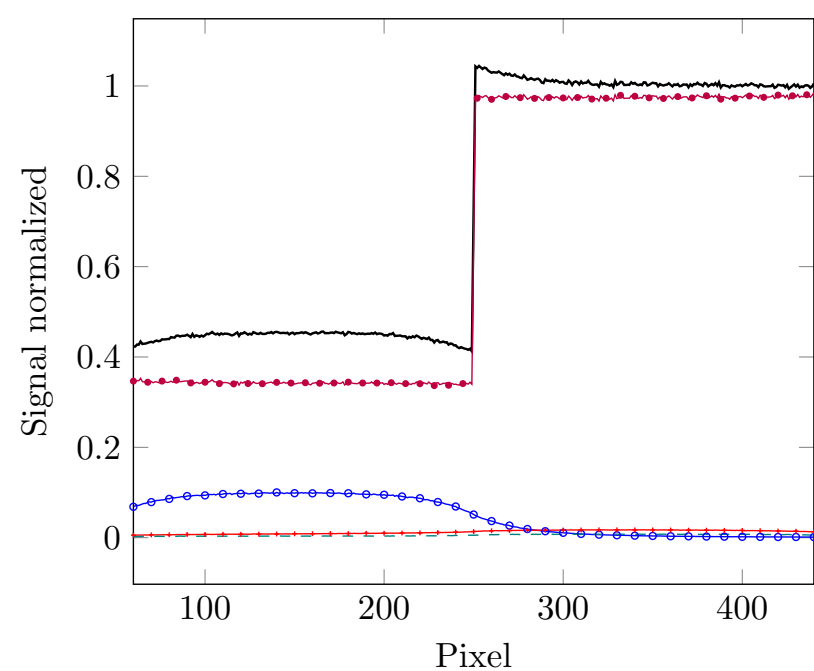

- Signal
- Signal without scattered radiation
- Object scattered radiation
- Detector internal scattering
-- Entrance window scattering

(a) Simulated edge spread function for the $10 \mathrm{~mm}$ edge object.

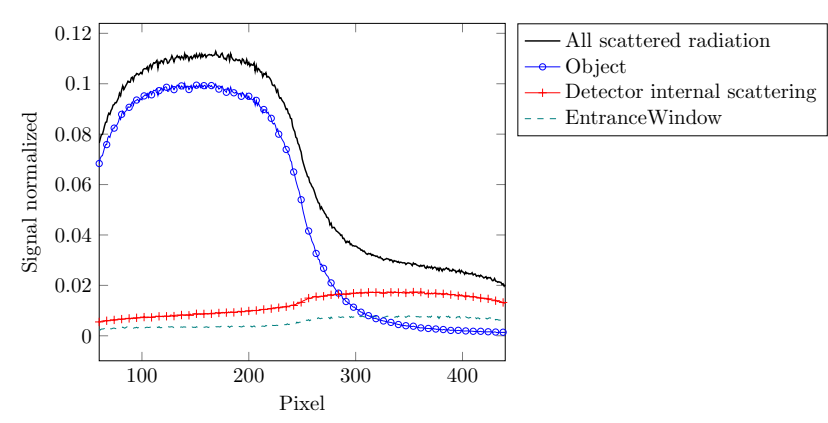

(b) Close-up of the simulated edge spread function on the scattered radiation.

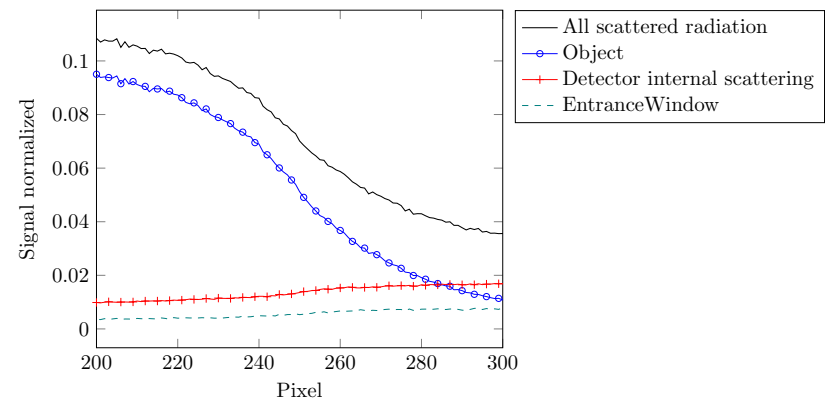

(c) Close-up of the simulated edge spread function on the scattered radiation at the edge.

Fig. 10: Figure 10a shows the results of the simulation of the edge spread function for the $10 \mathrm{~mm}$ edge object. The recorded photons were classified corresponding to the volume of scattering. Figure $10 \mathrm{~b}$ shows a close-up of the scattered radiation. Figure 10c shows the detailed scattered radiation around the edge.

[9] C. Stritt, P. Schuetz, M. Plamondon, A. Flisch, J. Hofmann, U. Sennhauser, Quantitative untersuchung der streubeiträge in hochenergie-röntgencomputertomografie, Materials Testing 58 (2) (2016) 122-126.

[10] P. R. Granfors, R. Aufrichtig, Performance of a $41 \times 41-\mathrm{cm} 2$ amorphous silicon flat panel $\mathrm{x}$-ray detector for radiographic imaging applications, Medical physics 27 (6) (2000) 1324-1331.

[11] U. Neitzel, S. Günther-Kohfahl, G. Borasi, E. Samei, Determination of the detective quantum efficiency of a digital $\mathrm{x}$-ray detector: comparison of three evaluations using a common image data set, Medical physics 31 (8) (2004) 2205-2211.

[12] E. Buhr, S. Günther-Kohfahl, U. Neitzel, Simple method for modulation transfer function determination of digital imaging detectors from edge images, in: Medical Imaging 2003, International Society for Optics and Photonics, 2003, pp. 877-884. 